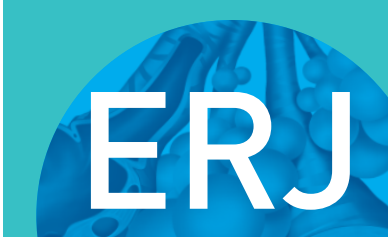

open research

\title{
High sensitivity of PD-L1 analysis from pleural effusion in nonsmall cell lung cancer
}

\author{
Lars Hagmeyer (1) ${ }^{1,2}$, Stephan Schäfer ${ }^{3}$, Marianne Engels³ ${ }^{3}$ Anja Pietzke-Calcagnile, \\ Marcel Treml ${ }^{1}$, Simon-Dominik Herkenrath ${ }^{1,2}$, Matthias Heldwein ${ }^{4}$, \\ Khosro Hekmat ${ }^{4}$, Sandhya Matthes $\mathbb{B}^{2}$, Andreas Scheel $\mathbb{B}^{3}$, Jürgen Wolf ${ }^{5}$, \\ Reinhard Büttner ${ }^{3}$ and Winfried Randerath (1) ${ }^{1,2}$
}

ABSTRACT Background: Programmed cell death protein 1 (PD-1)/programmed cell death protein ligand 1 (PD-L1) immune checkpoint inhibitors have been approved for monotherapy of metastatic nonsmall cell lung cancer (mNSCLC) depending on tumour cells' PD-L1 expression. Pleural effusion is common in mNSCLC. The significance of immunocytochemistry PD-L1 analysis from pleural effusion samples is unclear.

Aim: The aim of the study was to analyse the sensitivity regarding immunocytochemistry PD-L1 analysis of pleural effusion in NSCLC as compared to immunohistochemistry of pleural biopsies.

Patients and Methods: Fifty consecutive subjects (17 female, median age 72.5 years, seven neversmokers) were enrolled in this prospective controlled two-centre study. Inclusion criteria were pleural effusion, suspected or known lung cancer, indication for pleural puncture and thoracoscopy, and written informed consent. Immunocytochemistry and immunohistochemistry PD-L1 analyses were performed with the Dako-PDL1-IHC-22C3pharmDx assay. Analysis for sensitivity, specificity, and positive and negative predictive value was performed for PD-L1 detection from pleural effusion.

Results: 50 subjects underwent pleural puncture and thoracoscopy. Pathological diagnoses were lung cancer (48), lymphoma (1) and mesothelioma (1). Sensitivity, specificity, positive predictive value and negative predictive value of PD-L1-testing with expression $\geqslant 50 \%$ defined as positive were $100 \%$ (95\% CI $46-100 \%), 63 \%(36-84 \%), 45 \%(18-75 \%)$ and $100 \%(66-100 \%)$, and with expression $\geqslant 1 \%$ defined as positive $86 \%$ (56-97\%), 43\% (12-80\%), 75\% (47-92\%) and 60\% (17-93\%).

Conclusion: PD-L1 analysis in tumour-positive pleural effusion samples shows a very high sensitivity and negative predictive value, especially regarding PD-L1 expression levels $\geqslant 50 \%$ (European Medicines Agency approval). Negative results are reliable and help in the decision against a first-line checkpoint inhibitor monotherapy. However, a 1\% cut-off level (United States Food and Drug Administration approval) leads to a markedly lower negative predictive value, making other invasive procedures necessary (NCT02855281).

@ERSpublications

PD-L1 analysis in tumour-positive pleural effusion samples is characterised by a very high sensitivity and negative predictive value. Negative results seem very reliable and could thus help in the decision against a first-line checkpoint inhibitor therapy. https://bit.ly/2GPCLYS

Cite this article as: Hagmeyer L, Schäfer S, Engels M, et al. High sensitivity of PD-L1 analysis from pleural effusion in nonsmall cell lung cancer. ERJ Open Res 2021; 7: 00787-2020 [https:// doi.org/10.1183/23120541.00787-2020].

This article has supplementary material available from openres.ersjournals.com

This study is registered at www.clinicaltrials.gov with identifier number NCT02855281. Data and documents will not be available or shared.

Received: 1 Nov 2020 | Accepted: 5 Nov 2020

Copyright $\odot$ ERS 2021. This article is open access and distributed under the terms of the Creative Commons Attribution Non-Commercial Licence 4.0. 


\section{Introduction}

The programmed cell death protein 1 (PD-1) physiologically acts as an immune checkpoint receptor, enabling self-tolerance by T-cells in normal tissue. Unbound PD-1 allows the normal immune response by T-cells to occur. Binding of PD-1 to the ligands PD-L1 and PD-L2 suppresses the immune response. PD-L1 expression on tumour cells leads to activation of PD-1 and suppression of cytotoxic T-cell activity. The T-cell tolerance allows the tumour cells to avoid recognition and elimination by the immune system [1].

Early studies showed that immune checkpoint inhibitors had positive therapeutic effects in nonsmall cell lung cancer (NSCLC) patients with detectable PD-L1 expression [2]. Positive results could be demonstrated in treatment-naïve as well as in previously treated advanced NSCLC. There is probably a correlation between tumour PD-L1 expression and improved antitumor activity [3].

Pembrolizumab, Nivolumab and Atezolizumab are the available PD-1/PD-L1 immune checkpoint inhibitors which have been approved by the European Medicines Agency (EMA) and United States Food and Drug Administration (FDA) for the treatment of metastatic NSCLC (mNSCLC) stage IV according to the Union for international cancer control. Remarkably, EMA and FDA approvals differ for certain indications (supplementary table S1). Major differences are: a) the approval of pembrolizumab for first-line monotherapy in mNSCLC expressing PD-L1 with a tumour proportion score (TPS) $\geqslant 50 \%$ according to EMA and with a TPS $\geqslant 1 \%$ according to FDA, respectively; b) the approval of atezolizumab solely by the FDA for first-line monotherapy in mNSCLC with $\geqslant 50 \%$ of tumour cells expressing PD-L1.

Lung cancer is the second most common cancer and is the primary cause of cancer-related death in both men and women in the United States [4]. Currently, $80 \%$ of patients with lung cancer are given a diagnosis of primary NSCLC. Malignant pleural effusion is a common complication of advanced lung cancer. The presence of malignant pleural effusion indicates a poorer prognosis for patients with lung cancer and reduces their quality of life. Pleural effusion is a convenient clinical sample with important clinical diagnostic significance. It may be an alternative source providing useful information about the neoplasm's biology in terms of molecular genetic and immunopathological profile.

This prospective diagnostic pilot study was conducted to analyse the sensitivity regarding immunocytochemistry PD-L1 analysis of pleural effusion in NSCLC patients compared to the reference standard of PD-L1 immunohistochemistry.

The primary analysis goal was to test whether immunocytochemistry analysis of PD-L1 from pleural effusion has a relevant diagnostic value as compared to the immunohistochemistry analysis of pleural biopsies as the reference standard. In this analysis the specific approval status of checkpoint inhibitors in the EMA and the FDA region was considered.

\section{Material and methods \\ Patients}

At two tertiary care centres patients presenting with pleural effusion and suspected or known underlying lung cancer with indication for pleural puncture and thoracoscopy between October 2016 and November 2018 were considered for potential study inclusion. Exclusion criteria comprised given contraindications to undergo thoracoscopy [5] and any medical, psychological or other condition impairing the patient's ability to provide informed consent. Histopathological data from bronchoscopic tissue samples were available in 40 cases.

\section{Study design}

The trial was a prospective controlled pilot two-centre study. It was approved by the Ethics Commission of Cologne University's Faculty of Medicine and registered on www.clinicaltrials.gov (NCT02855281). All patients gave their written informed consent before study-specific data collection. All interventions were undertaken as part of clinical routine.

Affiliations: ${ }^{1}$ Institute of Pneumology, University of Cologne, Solingen, Germany. ${ }^{2}$ Hospital Bethanien Solingen, Clinic of Pneumology and Allergology, Center for Sleep Medicine and Respiratory Care, Solingen, Germany. ${ }^{3}$ University of Cologne, Institute of Pathology, Cologne, Germany. ${ }^{4}$ University Hospital Cologne, Clinic for Cardiac and Thoracic Surgery, Cologne, Germany. ${ }^{5}$ University Hospital of Cologne, Lung Cancer Group Cologne, Department I of Internal Medicine, Cologne, Germany.

Correspondence: Lars Hagmeyer, Hospital Bethanien Solingen, Clinic for Pneumology and Allergology, Center of Sleep Medicine and Respiratory Care, Aufderhöherstraße 169-175, 42699 Solingen, Germany. E-mail: lars.hagmeyeraklinik-bethanien.de 


\section{Sampling pleural cytology}

The patients underwent ultrasound-guided puncturing of the pleural cavity at the location of effusion. The excess pleural fluid was removed and collected for later cytological analysis by an independent pathology department (SS, ME, RB).

\section{Sampling pleural histology}

Histological pleural samples were obtained by pleural biopsy, primarily applying an awake single-incision medical video-assisted rigid thoracoscopy (Storz, Tuttlingen, Germany) under analgosedation using midazolam, disoprivane and pethidine. If this approach was not feasible, the patient underwent a surgical 2-4-ports video-assisted thoracoscopy under general anaesthesia. In either case, at least nine biopsies from the parietal pleura were taken. Where feasible, biopsies were sampled from the dorsocaudal, dorsal/ dorsoapical, ventral and diaphragmatic parietal pleura.

\section{Pathology}

All samples were sent in for routine pathological analyses. Microscope slides from paraffin-embedded tissue and cell blocks from cytocentrifugation samples were analysed. In cases where malignant tumour cells could be detected, subsequent immunocytochemistry or immunohistochemistry PD-L1 analysis was performed with the Dako PD-L1 IHC 22C3 pharmDx assay (Agilent, Santa Clara, CA, USA). PD-L1 expression was quantified by a 5-level score $[6,7]$. For the assessment of potentially therapy-relevant PD-L1 expression levels, two different threshold levels of PD-L1 expression were considered as per the differing approval status (TPS $\geqslant 50 \%$ and TPS $\geqslant 1 \%$ ).

Regarding detection of malignancy and PD-L1-positivity, results were defined as negative where the tests were not feasible or in case of inconclusive findings.

\section{Statistical analysis}

Results of continuous variables are expressed as median and quartiles 1 and 3 . The number of PD-L1-positive and PD-L1-negative cases for each sample type were used for calculation of sensitivity and specificity.

Secondary analyses comprised intra-individual comparative descriptive analyses of all pathological results from all patients to assess correlation between immunocytochemistry and immunohistochemistry results on a qualitative level. All analyses were performed using IBM SPSS Statistics ${ }^{\mathrm{TM}}$ for Windows (version 26.0; IBM Corp., Armonk, NY, USA).

\section{Results}

\section{Patients and diagnostic procedures}

Fifty patients were enrolled in this study. Their anthropometric data and smoking status are shown in table 1 . In 41 out of the enrolled 50 cases the disease was classified as NSCLC (adenocarcinoma $n=33$; squamous cell carcinoma $n=7$; large cell neuroendocrine carcinoma $n=1$ ), and nine patients showed other histological malignancy types (small cell lung cancer $n=4$; mixed carcinoma $n=3$; non-Hodgkin lymphoma $\mathrm{n}=1$; sarcomatoid mesothelioma $\mathrm{n}=1$ ).

Pleural puncture as well as thoracoscopy were performed in all 50 patients. No relevant peri-interventional complications have been documented. PD-L1 analysis of pleural effusion and pleural tissue was indicated in all samples with evidence of tumour cells, excluding cases with small cell lung cancer. Mainly due to

\section{TABLE 1 Anthropometric data and smoking status of 50 patients included}

\section{Sex}

Female

Male

Age years

Body mass index $\mathrm{kg} \cdot \mathrm{m}^{-2}$

Smoking status

Never-smoker

Ex-smoker

Current smoker
$17(34 \%)$

$33(66 \%)$

$72.5(62.8-76.3)$

$25.7(23.2-28.5)$

$7(14 \%)$

$28(56 \%)$

$15(30 \%)$

Data are presented as $\mathrm{n}(\%)$ or median (interquartile range). 


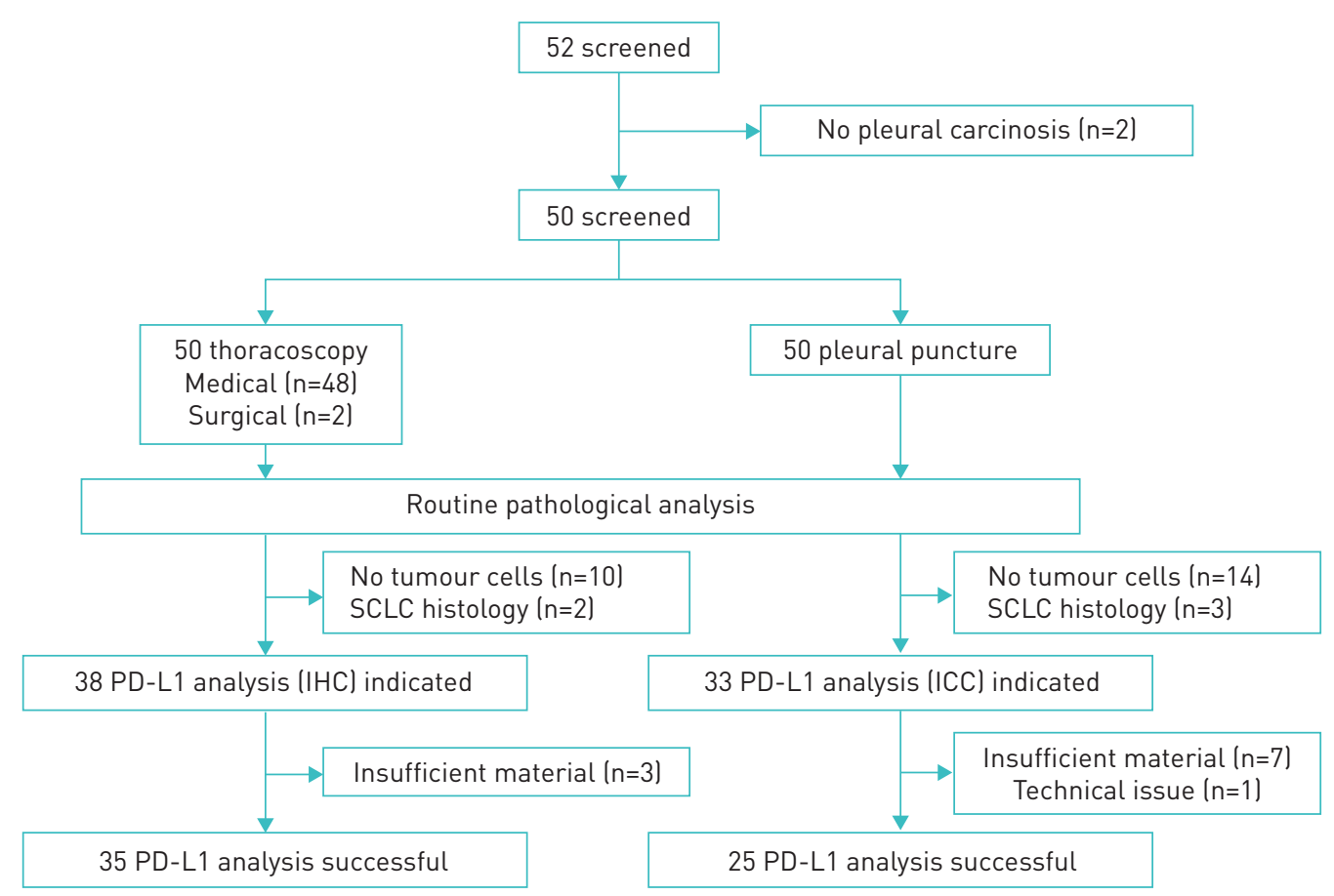

FIGURE 1 Flow chart depicting numbers of different samples and corresponding diagnostic procedures. PD-L1: programmed cell death protein ligand 1; SCLC: small cell lung cancer; IHC: immunohistochemistry; ICC: immunocytochemistry.

insufficient sample material (low tumour cell count), especially in pleural effusion, PD-L1 analysis could actually be performed in 25 (pleural effusion) and 35 (pleural tissue) cases (figure 1).

\section{Pleural histology, cytology and PD-L1 status}

The pathological evaluation of pleural effusion led to inconclusive results in seven cases, only describing cells with "suspicious" properties. The majority of cases where PD-L1 analysis was feasible showed PD-L1 expression with a TPS $\geqslant 1 \%$. In pleural effusion 13 of 25 cases (52\%, 95\% CI 34-70\%) were PD-L1-positive at a TPS $\geqslant 50 \%$, while in pleural tissue eight of 35 cases $(23 \%, 95 \%$ CI 12-39\%) were PD-L1-positive (table 2). A detailed breakdown of all cases regarding the detection of malignant tumour cells as well as PD-L1 status is given in the supplementary table S2.

Estimated sensitivity, specificity, and positive and negative predictive value of tumour cell detection according to immunocytochemistry analysis of pleural effusion compared to immunohistochemistry analysis of pleural biopsies were 83\% (95\% CI 67-89\%), 70\% (95\% CI 35-92\%), 92\% (95\% CI 76-98\%) and 50\% (95\% CI 24-76\%).

\section{Sensitivity and specificity of PD-L1 detection in pleural effusion based on all cases with} indication for $P D-L 1$ analysis

Based on all cases where PD-L1 analysis was indicated, the immunocytochemistry analysis of pleural effusion was compared with the immunohistochemistry analysis of pleural tissue (supplementary table S3).

\section{TABLE 2 Summary of malignancy and programmed cell death protein ligand 1 (PD-L1) status}

Pleural effusion $n$

\section{Cases}

Malignancy $(+/-/ O)$

PD-L1 $\geqslant 1 \%$ of tumour cells $(+/-/$ ND)

PD-L1 $\geqslant 50 \%$ of tumour cells $(+/-/ \mathrm{ND})$
50

$36 / 7 / 7$

$19 / 6 / 25$

$13 / 12 / 25$
Pleural biopsy $\mathbf{n}$

+: positive; -: negative; $\bigcirc$ : inconclusive; ND: not done. 


\begin{tabular}{|c|c|c|c|c|c|c|}
\hline & \multicolumn{3}{|c|}{$\begin{array}{l}\text { PD-L1 expression } \geqslant 50 \% \text { of tumour } \\
\text { cells defined as positive }\end{array}$} & \multicolumn{3}{|c|}{$\begin{array}{l}\text { PD-L1 expression } \geqslant 1 \% \text { of tumour } \\
\text { cells defined as positive }\end{array}$} \\
\hline & \multicolumn{2}{|c|}{ Pleural biopsy } & \multirow[t]{2}{*}{ Total } & \multicolumn{2}{|c|}{ Pleural biopsy } & \multirow[t]{2}{*}{ Total } \\
\hline & Positive & Negative & & Positive & Negative & \\
\hline \multicolumn{7}{|c|}{ Pleural effusion } \\
\hline Positive & 5 & 6 & 11 & 12 & 4 & 16 \\
\hline Negative & 0 & 10 & 10 & 2 & 3 & 5 \\
\hline Total & 5 & 16 & 21 & 14 & 7 & 21 \\
\hline
\end{tabular}

Two different alternatives were calculated: PD-L1 expression with a TPS $\geqslant 50 \%$ was defined as PD-L1-positive; and PD-L1 expression with a TPS $\geqslant 1 \%$ was defined as PD-L1-positive.

Estimated sensitivity and specificity of PD-L1 detection (PD-L1 expression with a TPS $\geqslant 50 \%$ defined as positive) according to immunocytochemistry analysis of pleural effusion compared to immunohistochemistry analysis of pleural biopsies were $71 \%$ (95\% CI 30-95\%) and $71 \%$ (95\% CI $49-87 \%)$. The positive predictive value was $42 \%$ (95\% CI 16-71\%) and the negative predictive value was $89 \%$ (95\% CI 65-98\%). The positive and the negative likelihood ratio was 2.45 (95\% CI 1.12-5.34) and 0.40 (95\% CI 0.12-1.35), respectively.

Estimated sensitivity and specificity of PD-L1 detection (PD-L1 expression with a TPS $\geqslant 1 \%$ defined as positive) according to immunocytochemistry analysis of pleural effusion compared to immunohistochemistry analysis of pleura biopsies were 71\% (95\% CI 44-89\%) and 64\% (95\% CI 36-86\%). The positive predictive value was $71 \%$ (95\% CI 44-89\%) and negative predictive value was $64 \%$ (95\% CI $36-86 \%)$. The positive and the negative likelihood ratio was 1.98 (95\% CI $0.92-4.26)$ and 0.46 (95\% CI $0.20-1.05)$, respectively.

\section{Sensitivity and specificity of PD-L1 detection in pleural effusion based on all cases with successful $P D-L 1$ analysis}

Based on all successful PD-L1 analyses (i.e. excluding samples with insufficient material for analysis or technical issues), the immunocytochemistry analysis of pleural effusion was compared with the immunohistochemistry analysis of pleural tissue (tables 3 and 4). Two different alternatives were calculated: PD-L1 expression with a TPS $\geqslant 50 \%$ was defined as PD-L1-positive.; and PD-L1 expression with a TPS $\geqslant 1 \%$ was defined as PD-L1-positive.

Estimated sensitivity, specificity, and positive and negative predictive value and likelihood ratios of PD-L1 detection according to immunocytochemistry analysis of pleural effusion compared to immunohistochemistry analysis of pleural biopsies are given in table 4 .

TABLE 4 Programmed cell death protein ligand 1 (PD-L1) detection in pleural effusion based on all cases with successful PD-L1 analysis. Sensitivity, specificity, positive predictive value, negative predictive value, positive likelihood ratio, negative likelihood ratio

\begin{tabular}{|c|c|c|c|c|}
\hline \multirow[t]{2}{*}{ Parameter } & \multicolumn{2}{|c|}{$\begin{array}{l}\text { PD-L1 expression } \geqslant 50 \% \text { of } \\
\text { tumour cells defined as } \\
\text { positive }\end{array}$} & \multicolumn{2}{|c|}{$\begin{array}{l}\text { PD-L1 expression } \geqslant 1 \% \text { of } \\
\text { tumour cells defined as } \\
\text { positive }\end{array}$} \\
\hline & Estimate & $95 \% \mathrm{Cl}$ & Estimate & $95 \% \mathrm{Cl}$ \\
\hline Sensitivity \% & 100 & $46-100$ & 86 & $56-97$ \\
\hline Specificity \% & 63 & $36-84$ & 43 & $12-80$ \\
\hline Positive predictive value $\%$ & 45 & $18-75$ & 75 & 47-92 \\
\hline Negative predictive value $\%$ & 100 & $66-100$ & 60 & $17-93$ \\
\hline Positive likelihood ratio & 2.67 & $1.42-5.02$ & 1.50 & $0.76-2.95$ \\
\hline Negative likelihood ratio & 0.00 & Not defined & 0.33 & $0.07-1.56$ \\
\hline
\end{tabular}


Sensitivity and negative predictive value were very high, and the negative likelihood ratio was very good with a PD-L1 expression TPS $\geqslant 50 \%$ defined as positive. For this cut-off value negative test results are robust and reliable, as they may allow more invasive diagnostic procedures to be dispensed with.

On the other hand, sensitivity and negative predictive value were lower with a PD-L1 expression TPS $\geqslant 1 \%$ defined as positive. For this cut-off value the test results may not be robust enough in the clinical decision-making process for or against a single agent first-line checkpoint inhibitor therapy.

For both cut-off values specificity is only moderate. This shows that there may be a relevant proportion of false-positive results and that positive test results from pleural effusion analysis need to be confirmed by more specific tests.

\section{Discussion}

This study could show that PD-L1 analysis from pleural effusion samples of NSCLC patients is an attractive diagnostic tool with a very high sensitivity and negative predictive value of up to $100 \%$.

In clinical routine immunocytochemistry-based PD-L1 analysis from malignant pleural effusion may be of great relevance. Particularly in patients with an increased risk of morbidity and mortality from invasive procedures such as thoracoscopy or bronchoscopy, the less invasive procedure of pleural puncture may allow a decision-making process for tumour-specific therapy.

With PD-L1 expression levels of TPS $\geqslant 50 \%$ defined as PD-L1-positive, it could be demonstrated that results from pleural effusion showed a high sensitivity (100\%), negative predictive value (100\%) and negative likelihood ratio $(0.00)$ with an acceptable specificity $(63 \%)$ and a limited positive predictive value (45\%). Given that pleural effusion was tumour cell-positive and PD-L1 analysis was feasible, no false negative results were documented. These statistical data suggest that negative results are highly reliable. The EMA approval for pembrolizumab as a single agent first-line therapy regimen was granted for patients with a TPS $\geqslant 50 \%$. Reflecting these considerations, it can be concluded that with negative PD-L1 analysis (TPS $<50 \%$ ) from pleural effusion samples a checkpoint inhibitor monotherapy is not feasible. The more invasive thoracoscopy will not add new information.

Considering these data, a clinical algorithm can be developed, which is shown in figure 2.

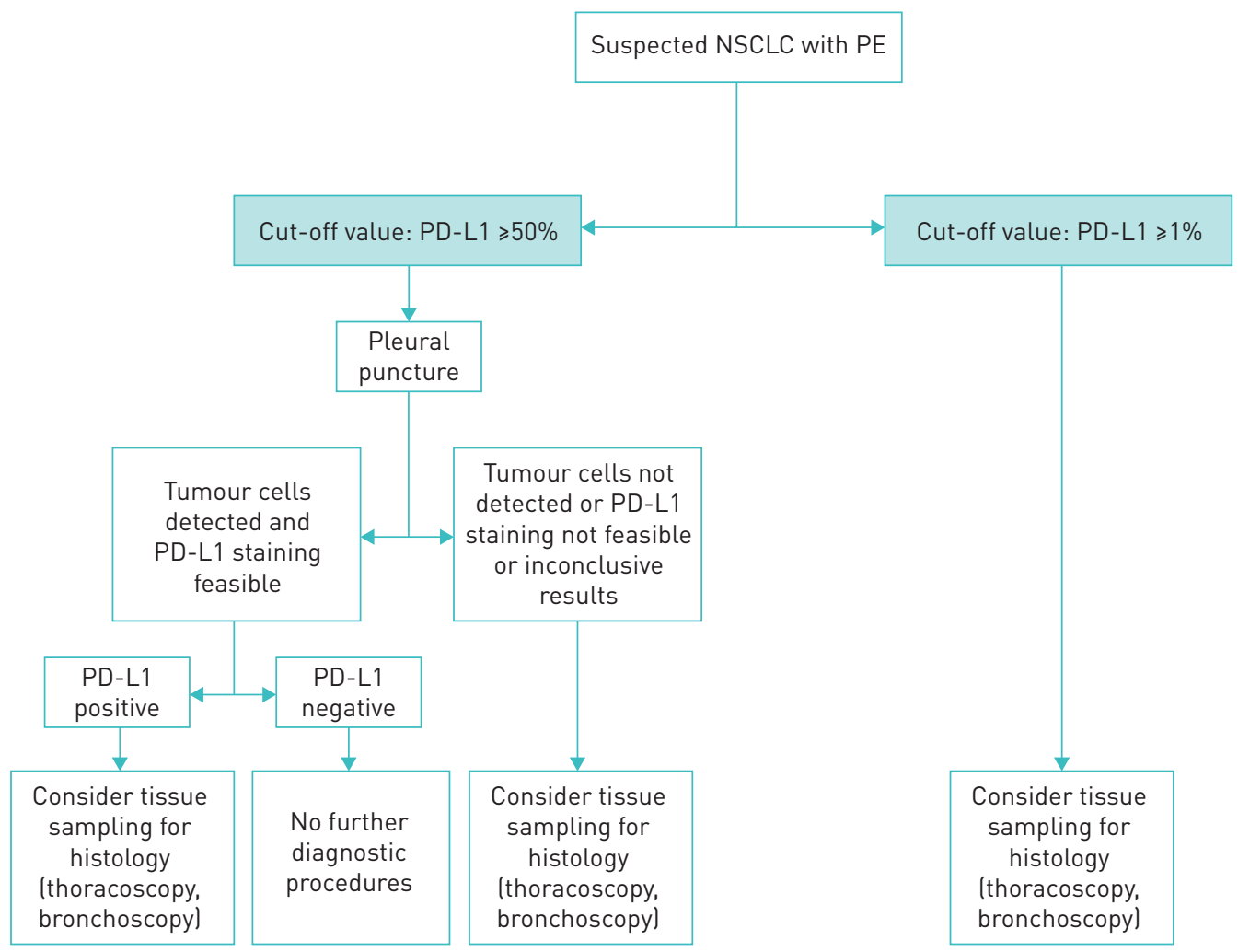

FIGURE 2 Proposed clinical algorithm for programmed cell death protein ligand 1 (PD-L1) testing in nonsmall cell lung cancer (NSCLC) patients with pleural effusion (PE). 
The same conclusions have to be drawn regarding the clinical indication and FDA approval of atezolizumab as a single agent first-line therapy.

However, tissue sampling by thoracoscopy or bronchoscopy should be considered when cytological analysis from pleural effusion does not detect tumour cells, when results remain inconclusive or when PD-L1-analysis is not feasible. This sequential approach is supported by the data from this study. Although results from pleural effusion samples were negative or inconclusive, PD-L1-positive tumour cells could be detected by thoracoscopy and/or bronchoscopy in some patients.

When PD-L1 expression levels with a TPS $\geqslant 1 \%$ were defined as PD-L1-positive, the sensitivity was good but lower $(86 \%)$ with an associated moderate positive predictive value $(75 \%)$, limited negative predictive value $(60 \%)$ and low specificity $(43 \%)$. The FDA approval was granted for pembrolizumab as a single agent first-line therapy with a TPS $\geqslant 1 \%$.

Under these conditions, dispensing with PD-L1 analysis should be considered in pleural effusion samples within the FDA-regulated region. Other invasive procedures should be favoured instead. Thoracoscopy or bronchoscopy may be more suitable to clarify the clinical indication for a single agent first-line immune checkpoint inhibitor therapy.

Overall, the data underlines the importance of applying appropriate diagnostic approaches in accordance with sensitivity and specificity values.

In some patients within this study, malignant cells were detected in pleural effusion and showed PD-L1 positivity, while the corresponding pleural tissue samples were free of malignancy. In clinical practice, such a constellation may prompt tumour tissue sampling from other sites in order to further substantiate the results from PD-L1 analysis.

To our knowledge this represents the first prospective study where sensitivity of PD-L1 analysis in pleural effusion has been determined. Overall, there is only limited evidence on the diagnostic significance of PD-L1 analysis in pleural effusion samples.

Grosu et al. [8] analysed a cohort of 82 subjects and reported a good correlation and concordance of PD-L1 results from pleural effusion and histological specimens with kappa values of 0.76 and 0.78 . However, due to the retrospective study design the data were heterogeneous as the samples were obtained under different clinical conditions. Some of the histological samples represented core biopsies from the primary tumour site, others represented material from surgical resections, whereas cytological specimens were obtained from pleural effusion. The time between histological and cytological sampling ranged from 0 to 363 days. At least one chemotherapy treatment was applied between surgical specimen collection and pleural effusion collection in $45 \%$ of patients. Due to the retrospective design, it cannot be ruled out that the results may be influenced by the different biological conditions of the neoplasm at the timepoint and location of tissue and pleural effusion sampling, respectively. In another retrospective study, 29 paired pleural effusion and tissue samples were analysed [9]. A history of chemotherapy was documented in $30 \%$ of the cases. With the limited number of samples and the given heterogeneity of the data conclusions have to be drawn with caution; however, the authors reported concordance between pleural effusion and histology results in 25 out of 29 cases. Zou et al. [10] retrospectively analysed the concordance of PD-L1 results in pleural effusion and histology samples obtained from various sites in 124 subjects. Although the PD-L1 expression was concordant in $86 \%$ of cases, a significant difference of expression levels was determined. This difference was reduced by a not yet validated TTF1 plus PD-L1 double staining protocol. Considering that two novel approaches have been performed in this study, the results need to be further substantiated. Another retrospective study analysed 51 cases by comparing PD-L1 results from pleural effusion and pleural biopsies using a novel PD-L1 scoring system [11]. The overall correlation was substantial; however, the differences were significant for high PD-L1 expression levels.

An accurate interpretation of PD-L1 immunocytochemistry remains essential. Particularly in the event of a low proportion of PD-L1-positive cells in immunocytochemistry analysis, it should be noted that false-positive results may be obtained, as staining of mesothelial cells can also occur, which cannot always be clearly distinguished from tumour cells outside a tissue context [12-14]. This may partially explain the limited specificity of PD-L1 analysis, especially when a TPS $\geqslant 1 \%$ is regarded as positive.

While the results of this study suggest that PD-L1 analysis from pleural effusion samples is not suited to completely replacing immunohistochemistry analysis of corresponding pleura tissue samples, it can be of additive value. The fact that the pleural effusion did not always yield enough material for a comprehensive immunocytochemistry evaluation, including PD-L1 analysis, constitutes an important limitation, in part leading to inconclusive results regarding malignancy. This may be due to a low rate of cells scaling off from the pleural lesion and/or a low rate of migration of tumour cells into the pleural fluid. 
As sensitivity and specificity analyses by their nature rely on the definition of a reference standard, we chose the immunohistochemistry analysis of pleural tissue for this purpose. In theory, as opposed to cytological preparations of pleural effusion, thoracoscopically obtained pleural tissue samples from suspected tumour sites should offer a higher yield of good quality sample material. Based on those, it should be possible to successfully characterise the specific features of each individual lung carcinoma, including PD-L1 expression. Tumour cells within the malignant pleural effusion can be assumed to have scaled off from pleural lesions, and thus their characteristics should closely resemble those of the histological samples. Thus, evaluating the results from immunocytochemistry analysis of pleural punctate compared to pleural tissue samples as the reference standard seems reasonable. On the other hand, a certain degree of tumour heterogeneity should be considered, which might cause differences in PD-L1 expression [15].

\section{Conclusion}

PD-L1 analysis in tumour-positive pleural effusion samples is characterised by a very high sensitivity and negative predictive value, especially regarding PD-L1 expression with a TPS $\geqslant 50 \%$. Negative results based on this cut-off seem very reliable and could thus help in the decision against a first-line checkpoint inhibitor monotherapy in the EMA region. Within the FDA-regulated region, however, this analysis might be less reliable, as the results are judged mainly based on a $1 \%$ cut-off level, which leads to a markedly lower negative predictive value. This indicates the necessity to perform corresponding immunohistochemistry analysis of pleural tissue, bearing in mind the costs of the associated therapy.

Further studies are necessary to enhance the confidence in our results, to further assess the role of PD-L1 analysis based on additional bronchoscopy and to investigate the potential issue of false positive immunocytochemistry results.

Author contributions: L. Hagmeyer: substantial contributions to conception and design, analysis and interpretation of data, drafting the article, and finalising the version to be published, taking responsibility for the integrity of the work as a whole, from inception to published article. S. Schäfer: acquisition of data, analysis and interpretation of data, revising the article critically for important intellectual content, final approval of the version to be published. M. Engels: acquisition of data, analysis and interpretation of data, revising the article critically for important intellectual content, final approval of the version to be published. A. Pietzke-Calcagnile: acquisition of data, analysis and interpretation of data, drafting statistics section and revising the article, final approval of the version to be published. M. Treml: acquisition of data, analysis and interpretation of data, drafting statistics section and revising the article, final approval of the version to be published. S-D. Herkenrath: acquisition of data, analysis and interpretation of data, drafting statistics section and revising the article, final approval of the version to be published. M. Heldwein: acquisition of data, analysis and interpretation of data, revising the article critically for important intellectual content, final approval of the version to be published. K. Hekmat: acquisition of data, analysis and interpretation of data, revising the article critically for important intellectual content, final approval of the version to be published. S. Matthes: acquisition of data, analysis and interpretation of data, revising the article, final approval of the version to be published. A. Scheel: acquisition of data, analysis and interpretation of data, revising the article critically for important intellectual content, final approval of the version to be published. J. Wolf: acquisition of data, analysis and interpretation of data, revising the article critically for important intellectual content, final approval of the version to be published. R. Büttner: acquisition of data, analysis and interpretation of data, revising the article critically for important intellectual content, final approval of the version to be published. W. Randerath: substantial contributions to conception and design, analysis and interpretation of data, drafting the article, and finalising the version to be published.

Conflict of interest: L. Hagmeyer reports grants from MSD Sharp \& Dohme GmbH, Haar, Germany, during the conduct of the study; and grants from AstraZeneca, from Roche, from Boehringer Ingelheim and from Pfizer, outside the submitted work. S. Schäfer reports personal fees from Boehringer Ingelheim and BMS outside the submitted work. M. Engels has nothing to disclose. A. Pietzke-Calcagnile has nothing to disclose. M. Treml has nothing to disclose S-D. Herkenrath has nothing to disclose. M. Heldwein has nothing to disclose. K. Hekmat has nothing to disclose S. Matthes has nothing to disclose. A. Scheel has nothing to disclose. J. Wolf reports personal fees from Abbvie, AstraZeneca and Blueprint, grants and personal fees from BMS, personal fees from Böhringer, Chugai and Ignyta, grants and personal fees from Jannsen, personal fees from Lilly and Loxo, grants and personal fees from MSD, Novartis and Pfizer, and personal fees from Roche and Takeda, outside the submitted work. R. Buettner has nothing to disclose. W. Randerath reports speaking fees and travel grants from Philips Respironics, Heinen and Löwenstein, Resmed, Bayer Vital, Bioprojet, and Vanda Pharma, outside the submitted work.

Support statement: This research was funded by MSD Sharp and Dohme GmbH, Haar, Germany. Funding information for this article has been deposited with the Crossref Funder Registry.

\section{References}

$1 \quad$ Poole RM. Pembrolizumab: first global approval. Drugs 2014; 74: 1973-1981.

2 Rizvi N, Mazières J, Planchard D. Safety and clinical activity of MK-2475 as initial therapy in patients with advanced nonsmall cell lung cancer (NSCLC). J Clin Oncol 2014; 32: Suppl. 15, 8007.

3 Garon E, Gandhi L, Rizvi N. LBA43 - Antitumor Activity of Pembrolizumab (Pembro; Mk-3475) and Correlation with Programmed Death Ligand 1 (Pd-L1) Expression in a Pooled Analysis of Patients (Pts) with Advanced NonSmall Cell Lung Carcinoma (Nsclc). Ann Oncol 2014; 25, Suppl. 5: v1-v41.

$4 \quad$ Siegel R, Naishadham D, Jemal A. Cancer statistics, 2013. CA Cancer J Clin 2013; 63: 11-30. 
5 Rahman NM, Ali NJ, Brown G, et al. Local anaesthetic thoracoscopy: British Thoracic Society Pleural Disease Guideline 2010. Thorax 2010; 65: Suppl. 2, ii54-ii60.

6 Scheel AH, Dietel M, Heukamp LC, et al. [Predictive PD-L1 immunohistochemistry for nonsmall cell lung cancer: Current state of the art and experiences of the first German harmonization study]. Pathologe 2016; 37: 557-567.

7 Scheel AH, Dietel M, Heukamp LC, et al. Harmonized PD-L1 immunohistochemistry for pulmonary squamous-cell and adenocarcinomas. Mod Pathol 2016; 29: 1165-1172.

8 Grosu HB, Arriola A, Stewart J, et al. PD-L1 detection in histology specimens and matched pleural fluid cell blocks of patients with NSCLC. Respirology 2019; 24: 1198-1203.

9 Song Z, Cheng G, Zhang Y. PD-L1 expression in malignant pleural effusion samples and its correlation with oncogene mutations in nonsmall cell lung cancer. J Thorac Dis 2020; 12: 1385-1392.

10 Zou Y, Xu L, Tang Q, et al. Cytology cell blocks from malignant pleural effusion are good candidates for PD-L1 detection in advanced NSCLC compared with matched histology samples. BMC Cancer 2020; 20: 344.

$11 \mathrm{Xu} \mathrm{J}$, Han X, Liu C, et al. PD-L1 expression in pleural effusions of pulmonary adenocarcinoma and survival prediction: a controlled study by pleural biopsy. Sci Rep 2018; 8: 11206.

12 Derakhshan F, Ionescu D, Cheung S, et al. Use of programmed death ligand-1 (PD-L1) staining to separate sarcomatoid malignant mesotheliomas from benign mesothelial reactions. Arch Pathol Lab Med 2020; 144: 185-188.

13 Humphries MP, McQuaid S, Craig SG, et al. Critical appraisal of programmed death ligand 1 reflex diagnostic testing: current standards and future opportunities. J Thorac Oncol 2019; 14: 45-53.

14 Wang H, Agulnik J, Kasymjanova G, et al. Cytology cell blocks are suitable for immunohistochemical testing for PD-L1 in lung cancer. Ann Oncol 2018; 29: 1417-1422.

15 McLaughlin J, Han G, Schalper KA, et al. Quantitative assessment of the heterogeneity of PD-L1 expression in non-small-cell lung cancer. JAMA Oncol 2016; 2: 46-54. 